\title{
Relationship between calcification, atherosclerosis and matrix proteins in the human aorta
}

\author{
Aleksandra Kuzan', Jerzy Wisniewski², Krzysztof Maksymowicz ${ }^{3}$, \\ Magdalena Kobielarz ${ }^{4}$, Andrzej Gamian", ${ }^{1,}$, Agnieszka Chwilkowska ${ }^{6}$
}

\begin{abstract}
${ }^{1}$ Department of Medical Biochemistry, Faculty of Medicine, Wroclaw Medical University, Wroclaw, Poland ${ }^{2}$ Central Laboratory of Instrumental Analysis, Wroclaw University of Science and Technology, Wroclaw, Poland

${ }^{3}$ Department of Forensic Medicine, Faculty of Medicine, Wroclaw Medical University, Wroclaw, Poland ${ }^{4}$ Department of Mechanics, Materials and Biomedical Engineering, Faculty of Mechanical Engineering, Wroclaw University of Science and Technology, Wroclaw, Poland

${ }^{5}$ Department of Immunology of Infectious Diseases, Hirszfeld Institute of Immunology and Experimental Therapy, Polish Academy of Sciences, Wroclaw, Poland

${ }^{6}$ Department of Molecular and Cellular Biology, Faculty of Pharmacy, Wroclaw Medical University, Wroclaw, Poland
\end{abstract}

\begin{abstract}
Introduction. Extracellular matrix (ECM) proteins have been associated with atherosclerotic complications, such as plaque rupture, calcification and aneurysm. It is not clear what role different types of collagen play in the pathomechanism of atherosclerosis. The aim of the study was to analyze the content of elastin and major types of collagen in the aortic wall and how they associated are with course of atherosclerosis.

Material and methods. In this work we present six biochemical parameters related to ECM proteins and collagen-specific amino acids (collagen type I, III, and IV, elastin, proline and hydroxyproline) analyzed in 106 patients' aortic wall specimens characterized by different degree of atherosclerosis. Liquid Chromatography Electrospray Ionization Tandem Mass Spectrometry (LC/ESI-MS/MS), ELISA and immunohistochemical methods were used. The severity of atherosclerosis was assessed on the six-point scale of the American Heart Association, taking into account the number and location of foam cells, the presence of a fatty core, calcium deposits and other characteristic atherosclerotic features. Results. The results show that there is a relationship between the content of collagen-specific amino acids and development of atherosclerosis. The degree of atherosclerotic lesions was negatively correlated with the content of proline, hydroxyproline and the ratio of these two amino acids. Calcium deposits and surrounding tissue were compared and it was demonstrated that the ratio of type I collagen to type III collagen was higher in the aortic tissue than in aortic calcification areas, while the ratio of collagen type III to elastin was smaller in the artery than in the calcium deposits. Conclusions. We suggest that increase in collagen type III presence in the calcification matrix may stem from disorders in the structure of the type I and III collagen fibers. These anomalous fibers are likely to favor accumulation of the calcium salts, an important feature of the process of atheromatosis. (Folia Histochemica et Cytobiologica 2021, Vol. 59, No. 1, 8-21)
\end{abstract}

Key words: atherosclerosis; calcification; collagen type I, III, IV; elastin; proline, hydroxyproline; ELISA; LC/ESI-MS/MS; IHC

Correspondence address: PhD Aleksandra Kuzan

Department of Medical Biochemistry,

Faculty of Medicine, Wrocław Medical University,

Chalubinskiego 10, Wroclaw, Poland

phone: 48717841379 , fax: 717840085

e-mail: aleksandra.kuzan@umed.wroc.pl

\section{Introduction}

The extracellular matrix (ECM) is a complex network of extracellular molecules, mainly proteins and polysaccharides, interacting with each other and in contact with the majority of cells. Collagen is a struc-

This article is available in open access under Creative Common Attribution-Non-Commercial-No Derivatives 4.0 International (CC BY-NC-ND 4.0) license, allowing to download articles and share them with others as long as they credit the authors and the publisher, but without permission to change them in any way or use them commercially. (c) Polish Society for Histochemistry and Cytochemistry 
tural and stable protein, with a long half-life span. This protein defines biomechanical properties of the tissues, e.g. tensile strength of arteries subjected to a high blood pressure. At the same time it is a protein that is being constantly modified and it is interacting with other tissue proteins. These features are important to providing homeostasis in the arteries and any alteration in that equilibrium can lead to the development of atherosclerosis. Contribution of collagen to the onset of atherosclerosis is complex. It includes formation of the scaffolds for modified lipoproteins, growth factors, advanced glycation end products, it is required for macrophage accumulation, proliferation and migration of smooth muscle cells or clot formation $[1,2]$. Collagen molecules are constantly undergoing degradation by matrix metalloproteases (MMPs), released among others by macrophages located in atheromatous plaques, weakening structure of the arteries. Its production may be stimulated or inhibited. Excessive metalloproteinases lead to atherosclerotic plaques being unstable, but their under-expression may lead to collagen accumulating in the form of fibrous caps, which has a significant impact on stiffening of the artery structure [2]. Twenty-eight types of collagen have been identified and described. The types I, III, and IV of collagen were selected here for their predominant presence and for functional importance in formation of the extracellular matrix.

Elastin is another key building block in the connective tissue, particularly important for the arteries' elasticity, allowing their return to original shape after stretching of the blood vessels. Such properties are determined by the presence of intramolecular desmosine bonds formed from three aldehyde derivatives of lysines with unchanged lysine residue. Elastin, in contrast to collagen, represents one genetic type; also, no elastin posttranslational modifications such as hydroxylation of lysines or glycosylation were observed [1].

There has been ongoing effort to establish what role specific types of collagen play and how the ECM proteins contribute to cardiovascular physiology and pathophysiology, particularly within the pathomechanism of atherosclerosis. The reports on ECM's role and on how the content of collagen and elastin in the arteries affects the severity of the atherosclerosis are inconclusive; it is not even clear whether the amount of collagen and elastin increases or decreases with the development of atherosclerosis [3-10]. The aim of this study was to define relationships between different types of collagen (I, III, IV) and elastin in arteries at various stages of atherosclerosis.

\section{Material and methods}

Human samples and their classification. The studied biological material consisted of 106 fragments of the abdominal or thoracic aorta sections collected during forensic and medical autopsies performed at the Department of Forensic Medicine of Wroclaw Medical University, Poland. Each sample was from a different patient. The samples came from people who died suddenly, aged $55 \pm 15$ years (mean \pm SD); $73 \%$ of them were men and $27 \%$ women. A formal requirement for the consent was met and the study was approved by the Bioethics Committee of the Wroclaw Medical University (No 220/2010). The probes were divided into six stages of atherosclerosis, following the American Heart Association scale of Atherosclerosis [11-13]. The classification was carried out independently by two Co-authors (A. K. and A. Ch.). The stages were defined as: I — early lesions, II — fatty streaks, III - pre-atheroma, IV — atheroma, V — fibroatheroma, VI - ruptured lesion, calcified lesion or fibrotic lesion. Samples were subdivided into those in which macroscopically visible calcification were observed $(\mathrm{n}=31)$ and to samples with no visible calcification $(n=49)$ (in the remaining 26 cases, there were no calcification data available, so they were not analyzed in this respect). Examples of an uncalcified aorta calcified aorta, and an isolated calcium deposit are shown in Figure 1. In the group of calcified samples, in 16 of them a large deposit of calcium was visible so that it was prepared and analyzed independently of the rest of the samples. Samples were subjected to qualitative immunochemical (IHC) staining and quantitative analysis by ELISA (enzyme-linked immunosorbent assay) and liquid chromatography combined with tandem mass spectrometry (LC-ESI-MS/MS). Reagents, unless indicated otherwise in the text, were purchased from POCH (Gliwice, Poland) and SigmaAldrich (Saint Louis, MO, USA).

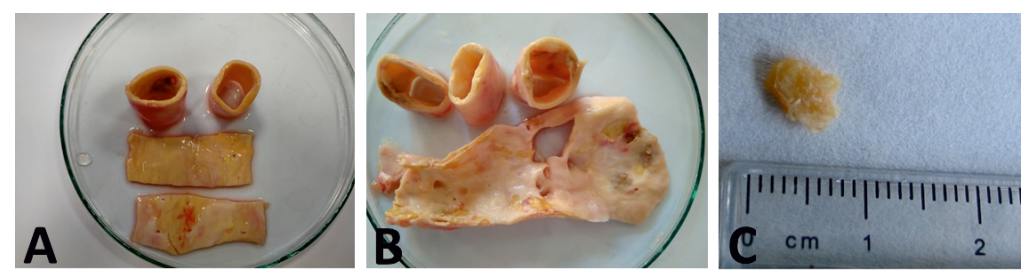

Figure 1. Macroscopic view of an uncalcified wall of aorta (A), a calcified wall of aorta (B) and the isolated calcium deposit (C). 
Quantification of collagens type I, type III, type IV and elastin content in aortas' fragments by ELISA. The samples about $5 \mathrm{~mm} \times 5 \mathrm{~mm} \times 3 \mathrm{~mm}$ were homogenized in extraction buffer (10 mM Tris, $5 \mathrm{mM}$ EDTA, $0.2 \mathrm{M} \mathrm{NaCl}$, pH 7.5) using a homogenizer FastPrep-24® (MP Biomedicals, Santa Ana, CA, USA) and the weight to buffer volume ratio of $100 \mathrm{mg} / 1 \mathrm{ml}$ was kept constant. The 96-well Maxisorp plates (Nunc ${ }^{\circledR}$, SigmaAldrich, Darmstadt, Germany) were coated with the homogenates. Standard solutions of collagen type I (Millipore, Billerica, MA, USA, cat. no.: CC050), collagen type III (Millipore, cat. no.: CC054), collagen type IV (Millipore, cat. no.: CC076), elastin (SigmaAldrich, cat. no.: E6902) were tested for the reference. The coating was carried out for $24 \mathrm{~h}$ at $4^{\circ} \mathrm{C}$. Next, the plates were blocked with $10 \%$ skim milk in PBST (PBS - phosphate buffered saline, $0.1 \%$ Tween 20 ) overnight at $4^{\circ} \mathrm{C}$. To detect collagens and elastin corresponding antibodies were applied: mouse monoclonal IgG1anti-collagen type I (Novus Biologicals, Abingdon, UK, clone COL1, cat. no.: NB600-450, 1:2000), mouse monoclonal anti-collagen type III (SigmaAldrich, clone FH-7A, cat. no.: C7805, 1:4000), mouse monoclonal anti-collagen type IV (SigmaAldrich, clone COL-94 cat. no.: C1926, 1:2000) or rabbit policlonal anti-elastin (Santa Cruz, Santa Clara, USA, clone H-300, cat. no.: sc-25736, 1:4000). After $2 \mathrm{~h}$ incubation secondary monoclonal anti-mouse IgG-HRP (H+L) (JacsonImmuno Research, West Grove, USA, cat. no.: 309-035-082, 1:5000) or anti-rabbit IgG-HRP $(\mathrm{H}+\mathrm{L})$ (JacsonImmuno Research, cat. no.: 111-035-045, 1:3000) was applied. After $1.5 \mathrm{~h}$ incubation the colorimetric reaction was developed using o-phenylenediamine (SigmaAldrich) and absorbance measured at $450 \mathrm{~nm}$ on EnSpire Multimode Plate Reader (Perkin Elmer, Waltham, MA, USA). The concentration of proteins in the homogenates was calculated from the standard curves obtained for individual standard solutions of collagens and elastin.

Immunohistochemistry. Tissue and isolated purified calcium deposit samples were fixed in $4 \%$ paraformaldehyde and embedded in paraffin. The paraffin blocks were cut into $5 \mu \mathrm{m}$ thick sections. They were next deparaffinized by immersing them in xylene, followed by washing the slides with a series of alcohol dilutions (100\%-50\% ethanol). To expose the antigen the slides were incubated with proteinase K (Dako, Santa Clara, CA, USA, cat. no.: S3020) for $10 \mathrm{~min}$ at $37^{\circ} \mathrm{C}$ and treated with Real Peroxidase-Blocking Solution (Dako, cat. no.: S2023) for 10 min and Protein Block (Dako, cat. no.: X0909) for 15 minutes. In the following step solutions of antibodies were applied: mouse monoclonal IgG1anti-collagen type I (Novus Biologicals, clone COL1, cat. no.: NB600-450, 1:2000), mouse monoclonal anti-collagen type III antibody (Santa Cruz, clone 3A1, cat. no.: sc-271249, 1:50), mouse monoclonal anti-collagen type IV (SigmaAldrich, clone COL94, cat. no.: C1926, 1:1000), mouse monoclonal anti-elastin (Santa Cruz, clone BA-4, cat. no.: sc-58756, 1:50), and slides incubated overnight at $4^{\circ} \mathrm{C}$ in a humid glass chambers. Finally, the immunocomplexes were visualized using the DAKO LSAB kit + System-HRP), successively with Biotynylated Link Universal, Streptavidin-HRP, DAB (diaminobenzidine) + substrate buffer with DAB + Chromogen, and following the general method guidelines. Delafield hematoxylin was used as the counterstain. Then the slides were immersed and closed with the glass coverslip using DPX (Aqua Medica, Lodz, Poland). The samples were analyzed with the light-field Olympus BX51 microscope (Olympus, Tokyo, Japan). For control of unspecific binding of secondary antibodies we made control incubations by omitting the primary antibody. These control experiments were negative.

Liquid chromatography electrospray ionization tandem mass spectrometry (LC-ESI-MS/MS) analysis. Lipids were removed from the aortic samples by incubating them in the mixture of $30 \mu \mathrm{L}$ of water and $300 \mu \mathrm{L}$ of methanol-chloroform $(2: 1 \mathrm{v} / \mathrm{v})$ and centrifugation at $15811 \mathrm{rpm}, 15 \mathrm{~min}, 4^{\circ} \mathrm{C}$. The precipitate was treated with $380 \mu \mathrm{L}$ methanol-chloroform-0.2 $\mathrm{M} \mathrm{HCl}$ (2:1:0.8), followed by centrifugation and lyophilization. Precipitate was suspended in $500 \mu \mathrm{L}$ of $6 \mathrm{M}$ $\mathrm{HCl}$ and incubated at $116^{\circ} \mathrm{C}$ for $18 \mathrm{~h}$. After the hydrolysis samples were dried with nitrogen and the precipitate was triturated with methanol, followed by drying with a stream of nitrogen. The resulting pellets were resuspended in $200 \mu \mathrm{L}$ of acetonitrile-water (1:1) containing $0.5 \mu \mathrm{g} / \mathrm{mL}$ glycylphenylalanine used as internal standard and centrifuged at $12000 \mathrm{rpm}\left(7 \mathrm{~min}, 20^{\circ} \mathrm{C}\right)$. The supernatant was filtered through $0.2 \mu \mathrm{m}$ syringe filters and then diluted 10 -fold with acetonitrile-water $(1: 1)$ to reduce the possibility of interfacing the analyte with the matrix. Quantitative measurements of proline (Pro) and hydroxyproline (ProOH) were made using a set of Acquity nanoUPLC Liquid Chromatographs equipped with Waters' Xevo G2 Q-TOF mass spectrometer (Waters, Milford, MA, USA). For the separation of samples, a HSS T3 column $(1 \times 50 \mathrm{~mm} ; 1.8 \mu \mathrm{m}$ particle size $)$ was used (Waters). The chromatographic separation was carried out at $80 \mu \mathrm{L} / \mathrm{min}$. The column was thermostated at $40^{\circ} \mathrm{C}$. The total chromatographic separation time, together with the time needed to regenerate the column, was $3 \mathrm{~min}$. The mobile phase components were $0.1 \%$ formic acid in acetonitrile (component A) and $0.1 \%$ formic acid in water (component B). Spectrometric measurements were performed using an electrospray ion source, in positive ionization mode, and in function MS/MS in high resolution. In order to ensure high accuracy of molecular mass readings, leucine enkephalin was used as internal standard. During the analysis of the fragmentation spectra, the characteristic daughter ions were sought: $70.06 \mathrm{~m} / \mathrm{z}$ for proline (parent ion: $116.07 \mathrm{~m} / \mathrm{z}$; collision energy: $12.5 \mathrm{eV}$ ); $86.06 \mathrm{~m} / \mathrm{z}$ for hydroxyproline (parent ion: $132.06 \mathrm{~m} / \mathrm{z}$, collision energy: $12.5 \mathrm{eV}$ ) and $120.08 \mathrm{~m} / \mathrm{z}$ for glycyl-L-phenylalanine (parent ion: $223.10 \mathrm{~m} / \mathrm{z}$, collision energy: $19 \mathrm{eV}$ ). 
Statistical analysis. The statistical analysis was performed using statistical package $\mathrm{R}$ for Windows (version 3.1.2) (R Core Team, Vienna, Austria). Correlations of individual parameters were calculated by Spearman correlation method. The Mann-Whitney test was used to investigate the association of individual atherosclerotic scales with protein content. Kruskall-Wallis test with post-hoc analysis of multiple repetitions with Bonferroni correction was used to analyze the association of atherosclerosis with all analyzed biochemical parameters. To compare the average protein content in calcium deposits and arteries, the Fisher test and Student's t-student tests were used. Wilcoxon test was carried out to compare paired data - calcium deposits and non-calcined parts from the same aorta. The analysis of the influence of sex on biochemical parameters was performed using the Mann-Whitney test, and the analysis of the difference between sex and severity of atherosclerosis was performed using a $\chi^{2}$ test. Significance in all cases was assumed at $p<0.05$.

\section{Results}

\section{Distribution of the studied ECM proteins in aortic wall}

Analysis of microscopic specimens with immunohistochemically stained collagen types and elastin (Figs. 2-5) showed localization of the studied proteins.
Type I collagen occurs in all three layers of the aortic wall in patients with lower stages of atherosclerosis, presenting fibers locating between cells (Fig. 2b, c). In samples that originated from people with more advanced atherosclerosis, type I collagen shows the tendency to concentrate, in some samples along the entire width of the artery (Fig. 2h) and in others in the inner layer of the artery (not shown). Similar localization and characteristics can be attributed to type III collagen (Fig. 3). In advanced atherosclerotic plaques type III and IV collagen was found in dense deposits in fibrous cap (Fig. $3 g$ and 4f, g).

In arteries collagen type IV is the major component of the basement membrane of vascular smooth muscle cells (VSMCs) and forms network connecting ECM collagens to these cells. The protein occurs both in the media and intima (Fig. 4). No collagen type IV was observed in tunica adventitia with an exception of the wall of smaller vessels (vasa vasorum) (Fig. 4a, b).

In aorta at stage I of atherosclerosis elastin forms strong and pronounced inner or/and outer elastic membranes (Fig. 5, b and c). In third stage of atherosclerosis, called pre-atheroma, and in IV stage of atherosclerosis, the width of the intima is strongly broadened and clear boundary between the intima and the media disappears, as a result of lack
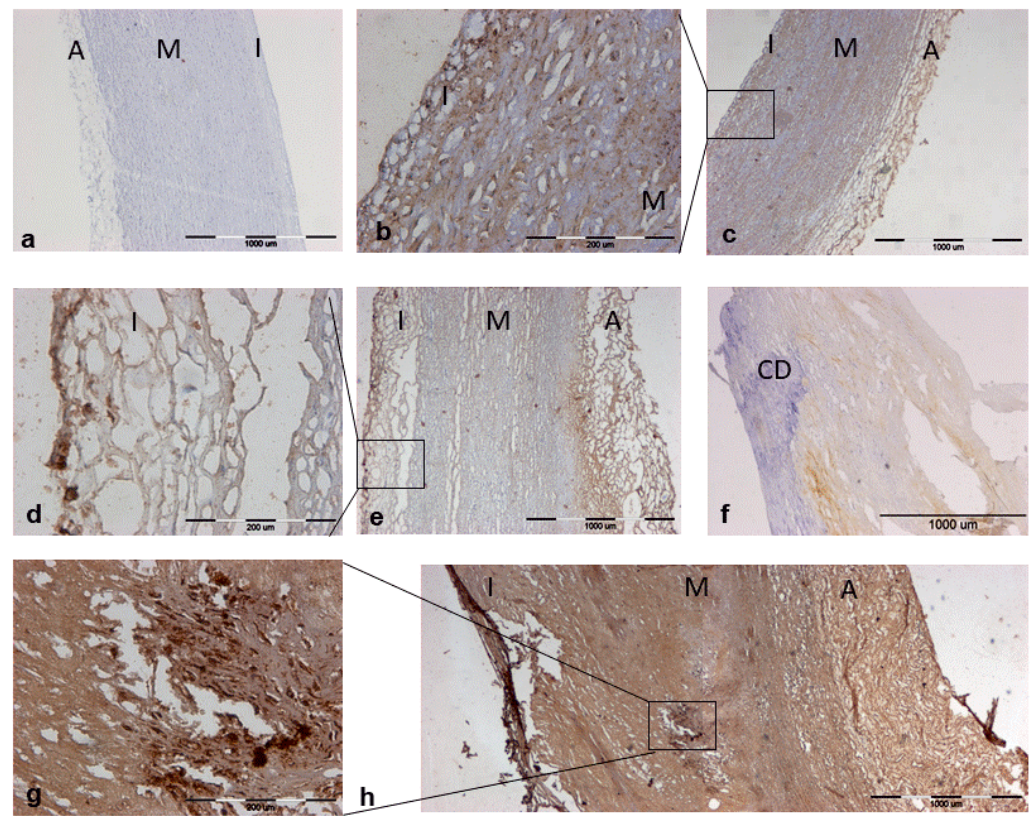

Figure 2. Localization of collagen type $\mathrm{I}$ in the wall of aortas at different stages of atherosclerosis. (a) Negative control without primary antibody, stage II; (b, c) Stage I of atherosclerosis; (d, e) III/IV stage of atherosclerosis; (f) calcium deposit; (g, h) V/VI stage of atherosclerosis. Collagen type I was detected by immunohistochemistry and sections were counterstained with hematoxylin (blue-purple) as described in Methods. Abbreviations: I — intima, M — media, A — adventitia, CD — calcium deposit. Scale bars: $1000 \mu \mathrm{m}$ (a, c, e, f, h) and $200 \mu \mathrm{m}$ (b, d, g). 

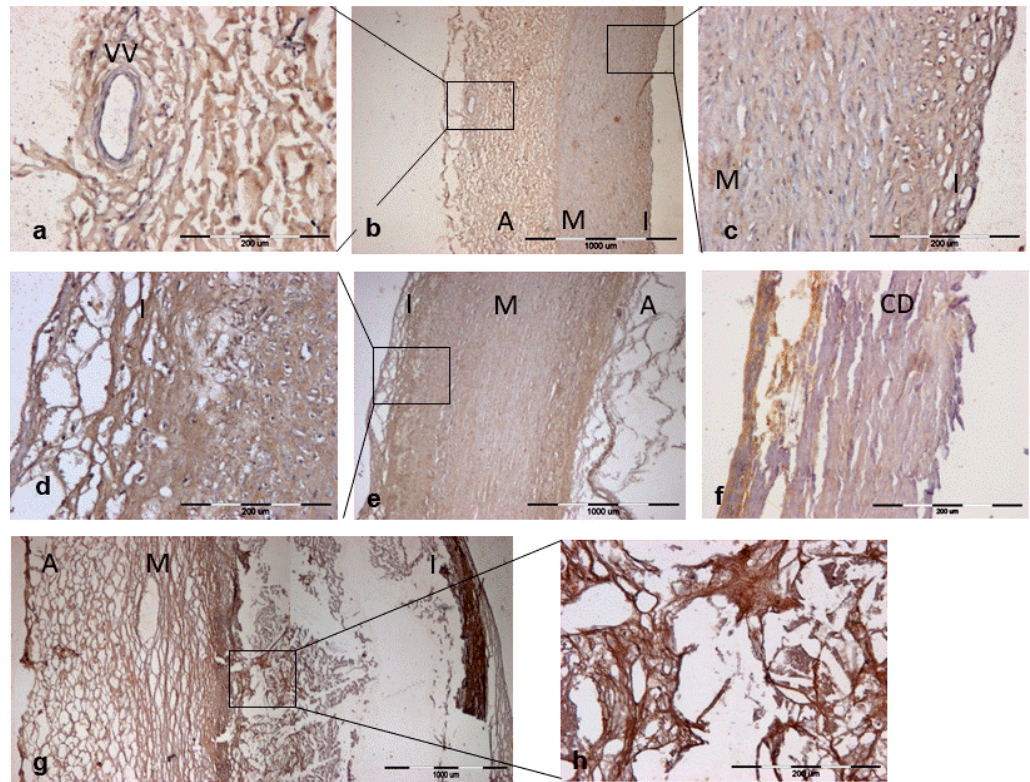

Figure 3. Localization of collagen type III in in the wall of aortas at different stages of atherosclerosis. Immunohistochemistry of representative sections with anti-collagen type III antibody (DAB, brown) and with hematoxylin counterstaining (blue-purple). (a-c) I stage of atherosclerosis; (d, e) III/IV stage of atherosclerosis; (f) calcium deposit; (g, h) V/VI stage of atherosclerosis. Abbreviations: I — intima, M - media, A — adventitia, CD - calcium deposit, VV — vasa vasorum. Scale bars $1000 \mu \mathrm{m}$ (b, e, f, g) and $200 \mu \mathrm{m}$ (a, c, d, h).
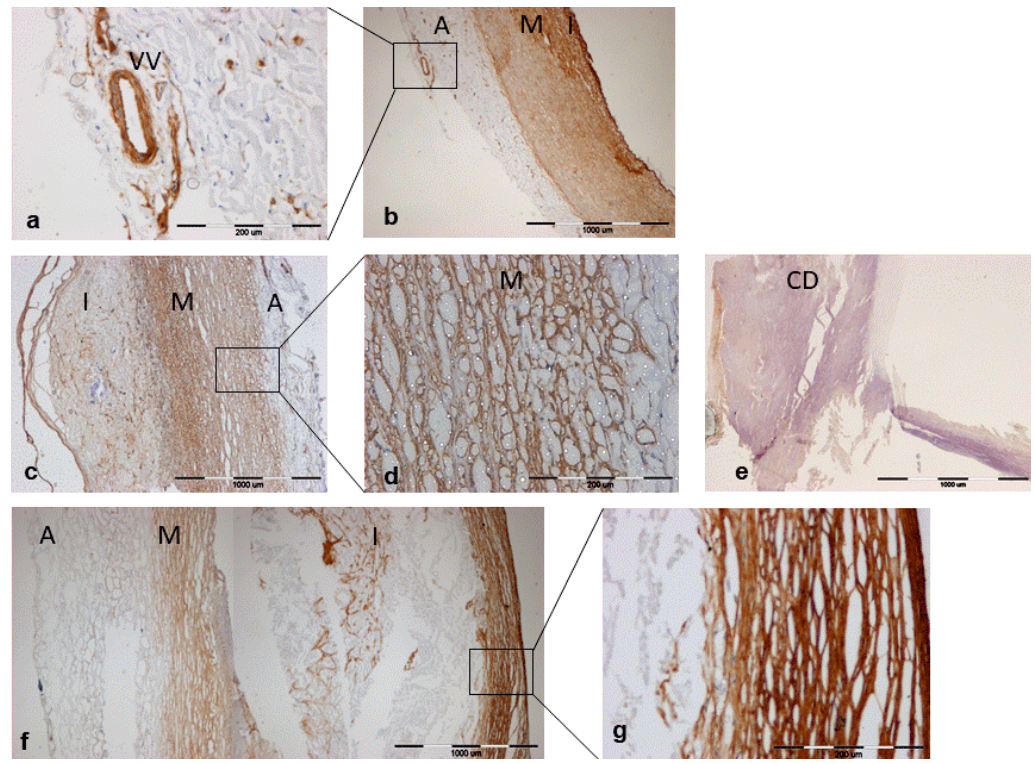

Figure 4. Localization of collagen type IV in the wall of aortas at different stages of atherosclerosis. Immunohistochemistry of representative sections with anti-collagen type IV antibody (DAB, brown) and with hematoxylin counterstaining (blue-purple). (a, b) I stage of atherosclerosis; (c, d) III/IV stage of atherosclerosis; (e) calcium deposit; (f, g) V/VI stage of atherosclerosis. Abbreviations: I — intima, M - media, A — adventitia, CD - calcium deposit, VV — vasa vasorum. Scale bars $1000 \mu \mathrm{m}$ (b, c, e, f) and $200 \mu \mathrm{m}(\mathrm{a}, \mathrm{d}, \mathrm{g})$.

of prominent internal elastic membrane (Fig. 5d). In more advanced stages of atherosclerosis elastin forms pseudomembranes passing between the cells, especially in the media (Fig. 5h, i).
Sections of aortic wall with calcium deposits (Fig. 2f, $4 \mathrm{e}, 5 \mathrm{f}$ ) show a trace presence of type I collagen, type IV collagen and elastin; however, type III collagen fibers (Fig. 3f) are present in a relatively large amount. 


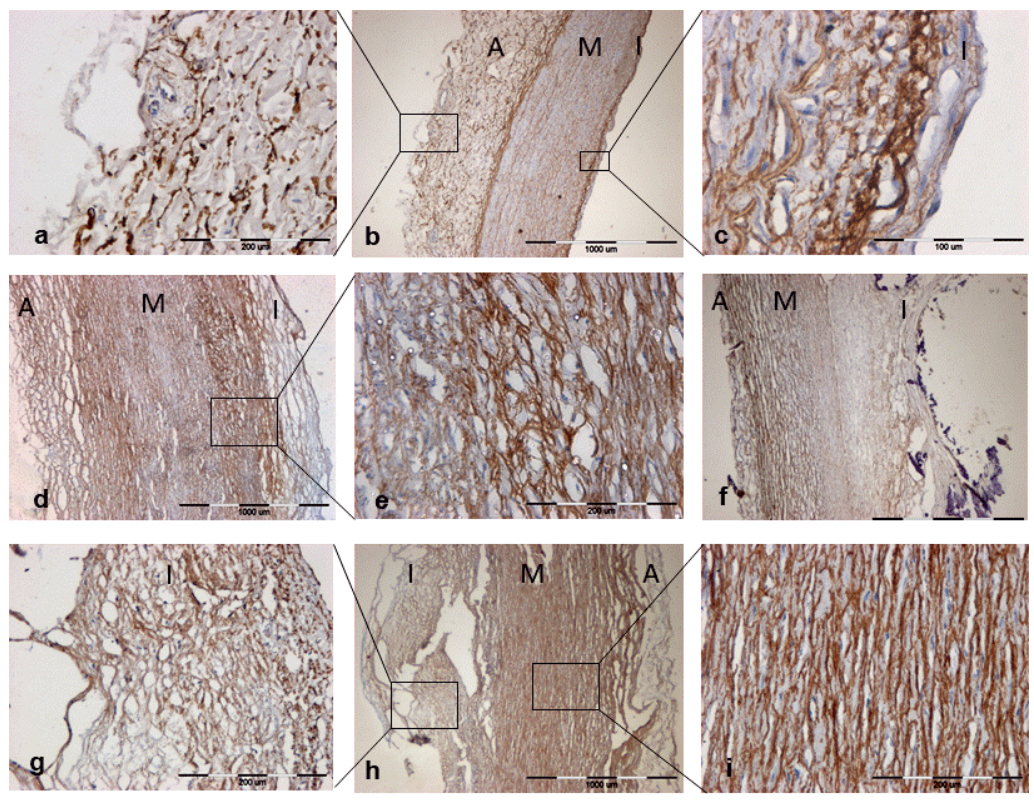

Figure 5. Localization of elastin in the wall of aortas at different stages of atherosclerosis. Immunohistochemistry of representative sections with anti-elastin antibody (DAB, brown) and with hematoxylin counterstaining (blue-purple). (a-c) I stage of atherosclerosis; (d, e) III/IV stage of atherosclerosis; (f) VI stage of atherosclerosis with space formed after preparation-related crushing of calcium deposit; (g-i) V/VI stage of atherosclerosis. Abbreviations: I - intima, M - media, A — adventitia. Scale bars: $1000 \mu \mathrm{m}$ (b, d, f, h), $200 \mu \mathrm{m}$ (a, e, g, i) and $100 \mu \mathrm{m}$ (c).

\section{Analysis of correlations between stage of atherosclerosis and biochemical parameters}

We have determined correlations between stage of atherosclerosis and the measured biochemical parameters: as well as between the content of type I and type III collagen, between type I and type IV collagen, between type I collagen and elastin, between type IV collagen and elastin and between type III and type IV collagens. All correlation coefficients were determined as positive and at medium strength (Table 1). We have also observed weak positive correlations between hydroxyproline and type I collagen content and hydroxyproline and total collagen content sum (Table 1).

Consequently, it was expected that the results obtained by ELISA and the LC-ESI-MS/MS methods would converge.

The proline and hydroxyproline concentration in aortic tissue was determined by liquid chromatography coupled with mass spectrometry (LC-ESI-MS/ MS), followed by statistical analysis with both qualitative (Spearman's correlation method, Table 1) and quantitative (Kruskall-Wallis test with repeated post-hoc analysis with Bonferroni correction, Table 2). There were statistically significant associations between degree of atherosclerosis and concentration in arterial tissue of proline content (very weak negative correlation, $r=-0.0436$ ), hydroxyproline (weak negative correlation, $r=-0.224$ ), proline to hydroxyproline ratio (weak negative correlation, $\mathrm{r}=-0.391$ ) and age (weak positive correlation, $r=-0.353$ ) (Table 1 ). These data indicate that as the disease progresses, the amino acids present in the collagen, and thus the protein content itself, fall. No statistically significant correlation was observed between the collagen types or elastin and degree of atherosclerosis (Table 1).

\section{Analysis of the influence of sex on biochemical parameters and advanced degree of atherosclerosis}

No relationship was observed between the gender and the content of the analyzed ECM proteins. Also, no difference was found between the degree of atherosclerosis between women and men (data not shown).

\section{Analysis of correlations between individual parameters}

We have determined a correlation between the following biochemical parameters, i.e. between type I and type III collagen, between type I and type IV collagen, between type I collagen and elastin, between type IV collagen and elastin and between type III and type IV collagens. All correlation coefficients were determined as positive and at medium strength (Table 1). We have also observed weak positive correlations between hydroxyproline and type I collagen content and hydroxyproline and collagen sum (Table 1). Consequently, it was expected that the results obtained by ELISA and the LC-ESI-MS/MS methods would converge. 
Table 1. Spearman correlation analysis between the studied biochemical parameters in aortic samples and between stage of atherosclerosis $(n=106)$ as well as between pairs of indicated parameters. Statistically significant results are marked by an asterisk. The levels of collagen type I, III, IV and elastin were measured by ELISA, and the levels of proline and hydroxyproline by LC-ESI-MS/MS.

\begin{tabular}{|c|c|c|}
\hline Pair of parameters & $\mathbf{r}$ & $\mathbf{p}$ \\
\hline Collage type I: collagen type III & 0.619 & $<0.0001^{*}$ \\
\hline Collagen type I: collagen IV & 0.437 & $<0.0001^{*}$ \\
\hline Collagen type I: elastin & 0.527 & $<0.0001^{*}$ \\
\hline Collagen type IV: elastin & 0.357 & $0.0002^{*}$ \\
\hline Collagen type III: collagen IV & 0.612 & $<0.0001^{*}$ \\
\hline Collagen type III: elastin & 0.543 & $<0.0001^{*}$ \\
\hline Collagen type I: hydroxyproline & 0.237 & $0.0259^{*}$ \\
\hline Collagen type III: hydroxyproline & 0.175 & 0.1046 \\
\hline Collagen type IV: hydroxyproline & 0.059 & 0.5822 \\
\hline Elastin: hydroxyproline & 0.109 & 0.322 \\
\hline Sum of the collagens: hydroxyproline & 0.214 & $0.0455^{*}$ \\
\hline Collagen type I: stage of atherosclerosis & 0.00918 & 0.9270 \\
\hline Collagen type III: stage of atherosclerosis & -0.178 & 0.0753 \\
\hline Collagen type IV: stage of atherosclerosis & -0.0915 & 0.3606 \\
\hline Elastin: stage of atherosclerosis & -0.043 & 0.6708 \\
\hline Proline: degree of atherosclerosis & -0.0436 & $<0.0001^{*}$ \\
\hline Hydroxyproline: degree of atherosclerosis & -0.224 & $0.0391^{*}$ \\
\hline Pro/ProOH ${ }^{\mathrm{a}}$ ratio:degree of atherosclerosis of atherosclerosis & -0.391 & $0.0002^{*}$ \\
\hline Age: degree of atherosclerosis & 0.353 & $0.0003^{*}$ \\
\hline
\end{tabular}

aPro, proline, ProOH, hydroxyproline

\section{Comparison of parameters between isolated calcium deposits and uncalcified aortic tissue}

The collagens and elastin contents in the sections of the arteries and in the calcium deposits were determined by ELISA and summarized in Table 3. It is noteworthy that in calcium deposits the determined values are several times lower than in arteries, although the percentage content of individual collagens and elastin for both groups of samples remains largely similar (Table 3 ). Differences in mean values between aortic walls and calcium deposits in the case of individual analyzed parameters are statistically significant except for the difference in the average collagen type III content (Table 3). The greatest difference is observed for type IV collagen - in calcium deposits its percentage is several times lower than in the artery.

\section{Comparison of parameters between calcified samples with and without signs of calcification}

In addition to the analysis of depleted calcium deposits, a division was also made between arterial samples based on whether they were calcified or not. We have found that the samples with calcifications are characterized by significantly lower proline content and ratio of proline to hydroxyproline (Table 4). Calcified samples were also derived from significantly older individuals and more advanced atherosclerosis than samples without calcification (Table 4). Comparison of calcium deposits with surrounding tissue revealed that calcium deposits had lower content of the extracellular matrix proteins (collagen type I, IV, elastin) and lower ratio of type I collagen to type III collagen (Table 5). However, in samples with calcium deposits three parameters are statistically higher than in surrounding tissues. They are: the ratio of collagen type I to elastin, the ratio of type III to type IV collagen and the ratio of type III collagen to elastin (Table 5).

\section{Discussion}

The structure of collagen and elastin shows a high degree of organization, which in turn determines the biological properties of these proteins. Even a small 
Table 2. Results of analysis differences between mean values of the analyzed parameters in aortic samples depending on the severity of atherosclerosis (Kruskall-Wallis test with post-hoc analysis of multiple repetitions with Bonferroni correction). Statistically significant results are marked by an asterisk. The levels of collagen type I, III, IV and elastin were measured by ELISA, and the levels of proline and hydroxyproline by LC-ESI-MS/MS.

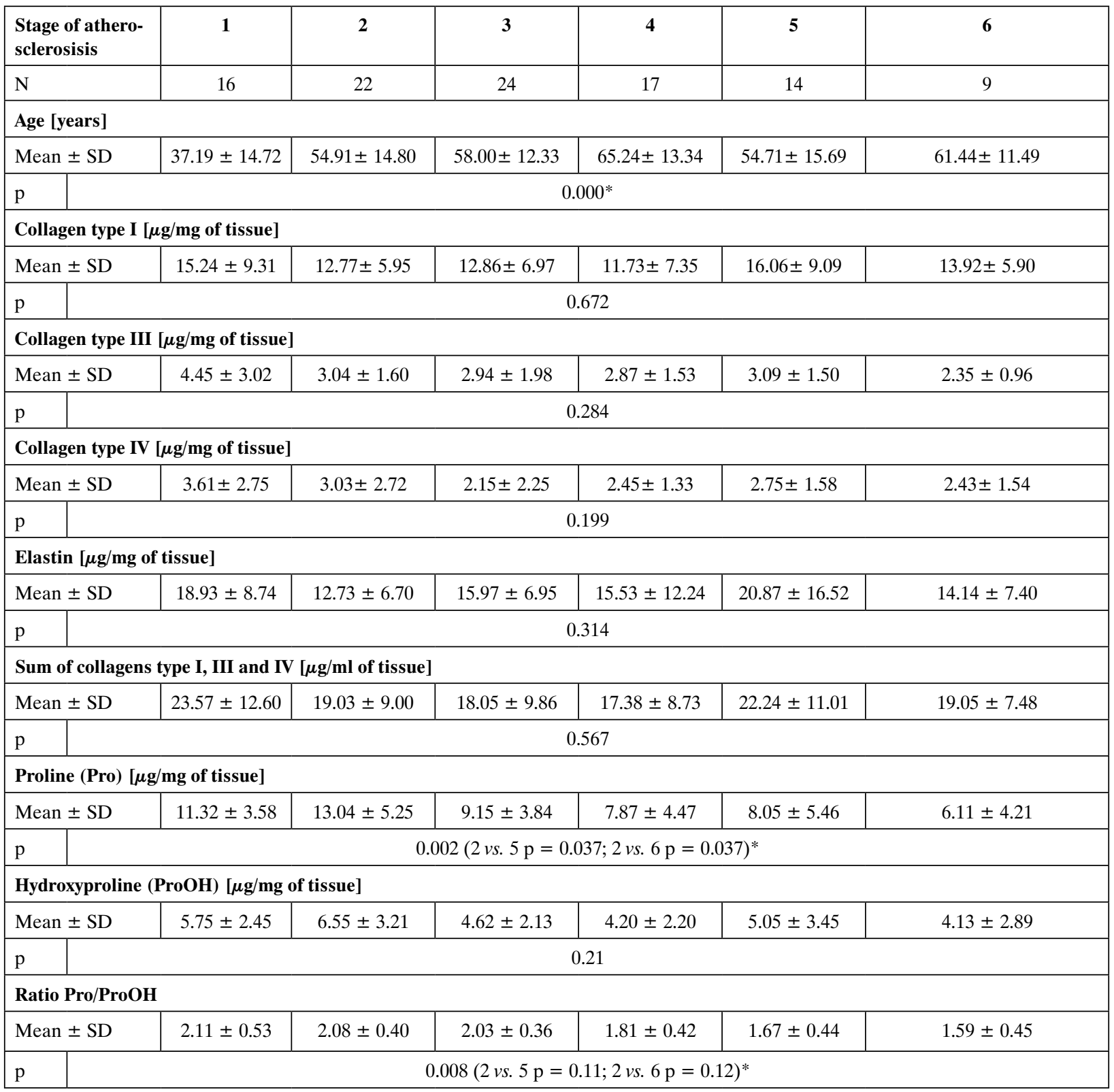

The results are mean \pm SD. The concentration of the studied compound was studied in aortic wall samples as described in Methods.

change in protein structure or in the expression of one of the ECM component, may change the ratio between individual proteins and lead to significant physiological disturbances. It has been postulated that especially aberrations in the structure and amount of collagen types I and III can result in atherosclerosis or hypertensive heart disease [14-17] while changes within the elastin fibers may lead to supraventricular stenosis, hypertension or aneurysm [14]. Our previous study referred to the role of collagen in the arteries in the context of glycation [18] and another one shows that there is a relationship between the content of type II collagen and the degree of atherosclerosis [19]. In the present study the object of interest are other types of fibrillar collagens, i.e. type I and type III, type IV collagen and elastin.

Progression of the atherosclerosis, the loss of elasticity and stiffness of the arterial walls, increases with age. These events are probably caused by increase in collagen content in the arteries and loss of elastin [20]. Unfortunately, this hypothesis has not been unequivocally verified. In the literature on the subject 
Table 3. Mean content of collagens type I, III, IV and elastin in the samples of aortic wall and in isolated calcium deposits. The levels of collagen type I, III, IV and elastin were measured by ELISA, and the levels of proline and hydroxyproline by LC-ESI-MS/MS.

\begin{tabular}{|c|c|c|c|c|c|}
\hline & \multicolumn{2}{|c|}{ Aorta $(n=106)$} & \multicolumn{2}{|c|}{ Calcium deposit $(n=16)$} & \multirow[t]{2}{*}{$p$} \\
\hline & $\begin{array}{c}\text { Content }[\mu \mathrm{g} / \mathrm{mg} \\
\text { of tissue] }\end{array}$ & $\begin{array}{c}\% \text { of the measured } \\
\text { proteins }\end{array}$ & $\begin{array}{c}\text { Content }[\mu \mathrm{g} / \mathrm{mg} \\
\text { of tissue] }\end{array}$ & $\begin{array}{c}\% \text { of the measured } \\
\text { proteins }\end{array}$ & \\
\hline Collagen type I & $13.3 \pm 7.44$ & 38.05 & $7.32 \pm 4.57$ & 45.47 & $0.000^{*}$ \\
\hline Collagen type III & $3.11 \pm 1.97$ & 8.90 & $2.29 \pm 1.51$ & 14.22 & 0.086 \\
\hline Collagen type IV & $2.69 \pm 2.17$ & 7.70 & $0.08 \pm 0.76$ & 0.50 & $0.000^{*}$ \\
\hline Elastin & $15.85 \pm 10.12$ & 45.35 & $6.41 \pm 7.36$ & 39.81 & $0.000^{*}$ \\
\hline Collagen to elastin ratio & \multicolumn{2}{|c|}{$1.21: 1$} & \multicolumn{2}{|c|}{$1.51: 1$} & \\
\hline
\end{tabular}

The results are mean \pm SD. The content of the studied compound was determined in aortic wall samples as described in Methods. Statistically significant results refer to the difference between arteries and calcium deposits are marked by an asterisk. 'Ratio of the sum of average collagens' content to average elastin content in aortic wall.

Table 4. Comparison of the patients' age, stage of atherosclerosis, content of extracellular matrix proteins and ratios of selected parameters between aortic samples without with calcium deposits.

\begin{tabular}{|l|c|c|c|}
\hline & $\begin{array}{c}\text { Samples without calcium } \\
\text { deposits }(\mathbf{n}=\mathbf{4 9})\end{array}$ & $\begin{array}{c}\text { Samples with calcium } \\
\text { deposits (n = 31) }\end{array}$ & $\boldsymbol{p}$ \\
\hline Age [years] & $49.56 \pm 15.07$ & $61.23 \pm 12.61$ & $0.00^{*}$ \\
\hline Stage of atherosclerosis [I-VI] & $2.44 \pm 1.29$ & $4.39 \pm 1.17$ & $0.00^{*}$ \\
\hline Collagen type I $\mu \mu \mathrm{g} / \mathrm{mg}$ of tissue] & $13.97 \pm 7.77$ & $14.06 \pm 7.97$ & 0.99 \\
\hline Collagen type III $[\mu \mathrm{g} / \mathrm{mg}$ of tissue] & $3.53 \pm 2.46$ & $2.78 \pm 1.28$ & 0.36 \\
\hline Collagen type IV $[\mu \mathrm{g} / \mathrm{mg}$ of tissue] & $2.90 \pm 2.39$ & $2.28 \pm 1.55$ & 0.41 \\
\hline Elastin $[\mu \mathrm{g} / \mathrm{mg}$ of tissue] & $15.57 \pm 8.53$ & $17.94 \pm 13.11$ & 0.66 \\
\hline Collagen type I/collagen type III ratio & $4.70 \pm 2.50$ & $5.39 \pm 2.11$ & 0.07 \\
\hline Collagen type I/collagen type IV ratio & $7.42 \pm 6.66$ & $9.48 \pm 9.79$ & 0.25 \\
\hline Collagen type I/elastin ratio & $1.69 \pm 4.22$ & $1.93 \pm 4.03$ & 0.48 \\
\hline Collagen type III/collagen type IV ratio & $1.67 \pm 1.24$ & $1.67 \pm 1.45$ & 0.83 \\
\hline Collagen type III/elastin ratio & $0.38 \pm 0.75$ & $0.34 \pm 0.66$ & $0.02^{*}$ \\
\hline Collagen type IV/elastin ratio & $0.41 \pm 1.33$ & $0.26 \pm 0.40$ & 0.14 \\
\hline Proline $[\mu \mathrm{g} / \mathrm{mg}$ of tissue] & $10.76 \pm 4.36$ & $8.17 \pm 5.17$ & $0.02^{*}$ \\
\hline Hydroksyproline $[\mu \mathrm{g} / \mathrm{mg}$ of tissue] & $5.65 \pm 2.72$ & $4.76 \pm 2.84$ & 0.26 \\
\hline Pro/ProOH ratio & $1.99 \pm 0.39$ & $1.69 \pm 0.42$ & $0.00^{*}$ \\
\hline
\end{tabular}

The results are mean $\pm \mathrm{SD}$. The content of the studied compounds is expressed as $\mu \mathrm{g} / \mathrm{mg}$ of tissue. Statistically significant results are marked by an asterisk.

there are contradicting reports, some are showing that with progression of the atherosclerosis collagen concentration increases [3-5], while the other reports describe decreased content of this protein $[6,21]$. Such large differences between the results described in the literature are probably due to the fact that each project analyzed a different study group using a variety of methods. It is likely that these differences are also the result of a statistical error related to too small size of a study group. Our study group was exceptionally large for a postmortem human study, so we conclude that the results are reliable. In the present study no particular collagen type has been demonstrated to correlate with atheromatosis (although the data suggests that one of the analyzed type - type III - may be associated with calcification, which sometimes accompanies atherogenesis). The data presented here indicate that there is a negative correlation between the hydroxyproline content, and thus collagen, and degree of atheromatosis. This is a novel observation, as no publication has been found that described the analysis of the content of proline and hydroxyproline in human aortas depending on the degree of atherosclerosis. Only a report by Abdelhalim et al. found 
Table 5. Comparative analysis of the content of the studied proteins in calcium deposits and surrounding tissue in samples taken from the same vascular wall.

\begin{tabular}{|c|c|c|c|}
\hline Parameter & & Mean \pm SD & $p$ \\
\hline \multirow{2}{*}{$\begin{array}{l}\text { Collagen type } \mathrm{I} \\
{[\mu \mathrm{g} / \mathrm{mg} \text { of tissue }]}\end{array}$} & Aorta $(\mathrm{n}=16)$ & $16.327 \pm 9.1889$ & \multirow[t]{2}{*}{$0.0093^{*}$} \\
\hline & Deposit $(n=16)$ & $6.854 \pm 4.5622$ & \\
\hline \multirow{2}{*}{$\begin{array}{l}\text { Collagen type III } \\
{[\mu \mathrm{g} / \mathrm{mg} \text { of tissue }]}\end{array}$} & Aorta $(\mathrm{n}=16)$ & $3.068 \pm 1.3926$ & \multirow[t]{2}{*}{0.065} \\
\hline & Deposit $(n=16)$ & $2.301 \pm 1.5976$ & \\
\hline \multirow{2}{*}{$\begin{array}{l}\text { Collagen type IV } \\
{[\mu \mathrm{g} / \mathrm{mg} \text { of tissue] }}\end{array}$} & Aorta $(\mathrm{n}=16)$ & $2.622 \pm 1.7534$ & \multirow[t]{2}{*}{$0.0021^{*}$} \\
\hline & Deposit $(n=16)$ & $0.885 \pm 0.7587$ & \\
\hline \multirow{2}{*}{$\begin{array}{l}\text { Elastin } \\
{[\mu \mathrm{g} / \mathrm{mg} \text { of tissue] }}\end{array}$} & Aorta $(n=16)$ & $23.682 \pm 14.6907$ & \multirow[t]{2}{*}{$0.0001^{*}$} \\
\hline & Deposit $(n=16)$ & $6.149 \pm 7.2043$ & \\
\hline \multirow[t]{2}{*}{ Collagen type I/III ratio } & Aorta $(\mathrm{n}=16)$ & $5.828 \pm 2.2741$ & \multirow[t]{2}{*}{$0.018^{*}$} \\
\hline & Deposit $(n=16)$ & $3.352 \pm 1.8551$ & \\
\hline \multirow[t]{2}{*}{ Collagen type $\mathrm{I} / \mathrm{IV}$ ratio } & Aorta $(n=16)$ & $11.108 \pm 12.4396$ & \multirow[t]{2}{*}{0.71} \\
\hline & Deposit $(\mathrm{n}=16)$ & $15.682 \pm 30.5617$ & \\
\hline \multirow[t]{2}{*}{ Collagen type I/elastin ratio } & Aorta $(\mathrm{n}=16)$ & $0.766 \pm 0.3757$ & \multirow[t]{2}{*}{$0.015^{*}$} \\
\hline & Deposit $(n=16)$ & $12.653 \pm 30.9017$ & \\
\hline \multirow[t]{2}{*}{ Collagen type III/IV ratio } & Aorta $(\mathrm{n}=16)$ & $1.701 \pm 1.8122$ & \multirow[t]{2}{*}{0.0042} \\
\hline & Deposit $(n=16)$ & $4.726 \pm 5.2757$ & \\
\hline \multirow[t]{2}{*}{ Collagen type III/elastin ratio } & Aorta $(\mathrm{n}=16)$ & $0.135 \pm 0.06409$ & \multirow[t]{2}{*}{0.0012} \\
\hline & Deposit $(n=16)$ & $4.667 \pm 8.2404$ & \\
\hline \multirow[t]{2}{*}{ Collagen type IV/elastin ratio } & Aorta $(\mathrm{n}=16)$ & $0.132 \pm 0.1144$ & \multirow[t]{2}{*}{0.3} \\
\hline & Deposit $(n=16)$ & $2.611 \pm 6.0602$ & \\
\hline
\end{tabular}

The result are mean $\pm \mathrm{SD}, \mathrm{n}=16$. Statistically significant results are marked by an asterisk.

a similar result in rabbits where animals on high fat diets had a reduced aortic hydroxyproline content [22]. Interestingly, although elastic fibers play a key role in the physiological functions of elastic type arteries such as aorta and pulmonary artery, we did not found correlation between elastin content in the wall of aorta and the progress of the atherosclerotic process. Other authors have reported both increased [7] and decreased $[1,8]$ elastin content in aortic wall during atherogenesis. Perhaps such ambiguous results are again the consequence of a statistical error related to too small size of a study group or individual differences in another study group, although our results indicate the simplest explanation - that there is no direct relationship between the elastin content and the severity of atherosclerosis.

Elastin and collagen have contrasting physical properties and the ratio of collagen to elastin should be of particular interest since it defines mechanical properties of the artery. According to Sakalihasan, the ratio of collagen to elastin in normal aortas is $1.85: 1$; however, it was shown to be higher in the wall of aortic aneurysms [23]. For our samples we have determined an average value of 1.21: 1 . In our study the statistical analysis did not show that this parameter for individual samples was related to a degree of atherosclerosis or age. Kong et al. [24] reported that ratio of elastin to collagen type III was increased in coronary arteries from patients with acute ischemic heart disease who had a myocardial infarction (the average age of patients with myocardial infarction was 57.4 years and did not differ statistically from the age of control patients). Perhaps the parameter which is the ratio of collagen to elastin is locally significant in specific manifestations of atherosclerosis [25]. For example, it is postulated that the ratio of collagen to elastin in porcine arteries is an accurate predictor of arterial burst pressure [26].

Our description of the distribution of collagens is consistent with earlier reports [27, 28]. Generally, the presence of collagen type I, III and IV was found in samples of a healthy aorta and atherosclerotic lesions, with fibrillar collagens occurring throughout the entire cross-section of aortic wall, and type IV collagen in intima and media as well as in the wall of vascular adventitious vessels. Collagens of all analyzed types have concentrated in the outer layer, forming fibrous cap above highly advanced plaques. In the fat core 
itself, however, a decrease in both fibrillar collagen and type IV collagen was observed.

In our project, we also analyzed the phenomenon of calcification. It is a clinically very serious problem that is complex in terms of etiology. It is reported that the formation of calcium deposits occurs in association with dyslipidemia, hypertension, uremia, glycation associated with diabetes, dysregulated mineral metabolism particularly hyperphosphatasemia, chronic oxidative stress, and is associated with a change in expression of fetuin-A and osteoprotegerin. We analyzed the relationship between aortic calcification and selected extracellular matrix proteins composition $[29,30]$.

Our research shows that in the deposits calcium salts displace organic components therefore ECM protein content in calcium deposits is lower than in soft parts of the arteries what is consistent with our observation. There is generally less proteins in calcium deposits than in the wall of the vessel from which they have been isolated. This is especially true for collagen type I, IV and elastin. Noteworthy is the small difference between the contents of type III collagen in the calcium deposits and in the surrounding tissue. In the deposits, there was also a lower ratio of type I to type III collagen. Microscopic images of the calcium deposit show a prominently higher immunoreactivity of type III collagen fibers compared to trace quantities of elastin, collagen I and IV. Calcium deposits show higher than the surrounding tissue ratio of type I collagen to elastin, type III collagen to type IV collagen, and type III collagen to elastin. It is somewhat intriguing, that for most of the aforementioned parameters that differentiate deposits and surrounding layer, when collagen type III content appears in the denominator, the parameter is higher in the artery, whenever it is present in the numerator, the parameter is lower in the artery than in the calcium deposit. It is also worth mentioning that type III collagen is the only protein of which content is not statistically lower in calcium deposit than in the soft part of the artery. The data point into some relationship between occurrence of this protein and calcification. The collagen type III seems to play a structural role in the deposit and its content exceeds that of the elastin, collagen type I and IV. There have been no other studies describing the role of type III collagen in the calcification of arteries, except for one by Kuga et al. [31] asserting that calcification in the abdominal aortic aneurysms was most likely associated with the breakdown of collagen type III fibrils [31]. There are few premises that collagen of this type may have a role in calcification, as it does in other tissues, such as bones and tendons [32,33]. As in this study, the results obtained by Lui et al. in a calcified tendinopathy model show that the level of collagen in calcified tendon seems to be decreasing, while the ratio of type III to type I collagen is increasing in this tissue [32]. It is well known that type I collagen is the main component of both arteries and tendons. Type III collagen is a protein incorporated in fibrils created by type I collagen. These authors hypothesized that the increase in collagen type III content in tissue undergoing calcification can be linked to abnormalities in the fiber structure - abnormalities that favor accumulation of calcium salts in that area [32]. It is possible that analogous processes govern arterial calcification.

Volk [33] drew attention to the potential impact that type III collagen may have on the osteoblasts differentiation. It is known that apart from soft tissues, high expression of this type of collagen is observed during skeletal embryonic development, and it is also synthesized by osteoblasts in mature bones [34]. When Col3a1 gene was silenced in mice, i.e., type III collagen gene was not expressed, the onset of the Ehlers-Danlos syndrome (EDS) was observed with functional impairment of the soft tissues, disturbed healing, and possibly the bone disorders [35]. It has been found that type III collagen plays a role in the differentiation of osteoblasts, thus conditioning the development of the spongy bone [33]. It is likely that this type of collagen contributes to arterial calcification by differentiating cells from osteoblast-like cells. However, to validate this hypothesis further research is required.

The guiding hypothesis for this research project was that there may be a link between the elastin content and calcification. Such assumption comes from literature reports describing formation of calcium deposits along elastin layers in the wall of an artery (membrana elastica interna and membrana elastica externa) [36]. This hypothesis is further supported by the reports showing that elastin metabolites can activate calcium deposition in smooth muscle cells [37]. The role of elastin in vascular calcification has also been demonstrated by several other authors in animal models [38-40]. However, the analysis of the data obtained in this study has not shown a statistically significant relationship between the content of elastin, or the ratio of this protein to other ECM components, and the presence of calcification foci. Immunohistochemical staining of the aortic sections also did not show a link between the location and organization of elastin and calcification. Therefore, our results do not support the thesis that elastin plays a causative role in the process of calcium deposition in blood vessels. The lack of such 
statistically significant evidence might be related to scarcity of calcified samples and their diversity (among artery segments were samples representing different types of calcification: accompanying atherosclerosis, Mönckeberg's arteriosclerosis, elastocalcinosis).

Summarizing, the extracellular matrix of the human aorta consists of nearly $45 \%$ of elastin and $55 \%$ of collagen, of which the majority are fibrillary collagens type I and III, occurring in all three layers of the artery wall. Type IV collagen accounts for nearly $10 \%$ of all abdominal aortic collagen, located in subendothelial basement membrane and also the basement membrane of VSMCs. Quantities of collagen types I, III and IV as well as elastin correlate positively with other analyzed proteins of extracellular matrix. However, in the study described here, there is no relationship between the content of collagen type I, III, IV or elastin and the degree of atherosclerosis. Noteworthy, the weak negative correlation between atherosclerosis and the amount of hydroxyproline is noted, indicating a loss of collagen with the onset of atherogenesis. The results of particular interest are those describing the role of ECM proteins in arterial calcification, and collagen type III playing both structural and regulatory role.

Since patients with the cardiovascular diseases, particularly those caused by atherosclerosis, experience high mortality, there is a pressing need to thoroughly investigate how degenerative lesions in the arteries are being formed. Further studies are also needed to resolve contradictory reports in the literature and unresolved question concerning the role that extracellular matrix proteins may have in atherogenesis and the interplay between various ECM components. Such studies may have a diagnostic value and there are already reports proposing the use of magnetic resonance imaging, positron emission tomography (PET) or single photon emission computed tomography (SPECT) [41, 42] in depicting the ECM changes. According to Järveläinen, Kassam, Zheng and others $[14,43-45]$ it is possible to influence pharmacologically the matrix protein content in the walls of the blood vessels. Therefore, such diagnostic and therapeutic tools would become indispensable in monitoring the progress of the treatment of cardiovascular diseases.

\section{Funding}

This publication is part of project „Wrovasc - Integrated Cardiovascular Center", co-financed by the European Regional Development Fund, within Innovative Economy Operational Program, 2007-2013 realized in Provincial Specialized Hospital, Research and Development Centre in Wroclaw.

\section{Acknowledgements}

We thank Dr. Elzbieta Gamian for preparing paraffin blocks.

\section{References}

1. Xu J, Shi GP. Vascular wall extracellular matrix proteins and vascular diseases. Biochim Biophys Acta. 2014; 1842(11): 2106-2119, doi: 10.1016/j.bbadis.2014.07.008, indexed in Pubmed: 25045854.

2. Rekhter M. Collagen synthesis in atherosclerosis: too much and not enough. Cardiovasc Res. 1999; 41(2):376-384, doi: 10.1016/ s0008-6363(98)00321-6, indexed in Pubmed: PMID: 10341837.

3. Lan TH, Huang XQ, Tan HM. Vascular fibrosis in atherosclerosis. Cardiovasc Pathol. 2013; 22(5): 401-407, doi: 10.1016/j. carpath.2013.01.003, indexed in Pubmed: 23375582.

4. Geary RL, Wong JM, Rossini A, et al. Expression profiling identifies 147 genes contributing to a unique primate neointimal smooth muscle cell phenotype. Arterioscler Thromb Vasc Biol. 2002; 22(12): 2010-2016, doi: 10.1161/01. atv.0000038147.93527.35, indexed in Pubmed: 12482827.

5. Chiong T, Cheow ESH, WooCC, et al. Aortic wall extracellular matrix proteins correlate with syntax score in patients undergoing coronary artery bypass surgery. Open Cardiovasc Med J. 2016; 10: 48-56, doi: 10.2174/1874192401610010048, indexed in Pubmed: 27347220.

6. Libby P, Aikawa M. Stabilization of atherosclerotic plaques: new mechanisms and clinical targets. Nat Med. 2002; 8(11): 1257-1262, doi: 10.1038/nm1102-1257, indexed in Pubmed: 12411953.

7. Foote CA, Castorena-Gonzalez JA, Ramirez-Perez FI, et al. Arterial stiffening in western diet-fed mice is associated with increased vascular elastin, transforming growth factor $\beta$, and plasma neuraminidase. Front Physiol. 2016; 7: 285, doi: 10.3389/fphys.2016.00285, indexed in Pubmed: 27458385.

8. Asciutto G, Dias NV, Edsfeldt A, et al. Low elastin content of carotid plaques is associated with increased risk of ipsilateral stroke. PLoS One. 2015; 10(3): e0121086, doi: 10.1371/journal. pone.0121086, indexed in Pubmed: 25803692.

9. Shami A, Gonçalves I, Hultgårdh-Nilsson A. Collagen and related extracellular matrix proteins in atherosclerotic plaque development. Curr Opin Lipidol. 2014; 25(5): 394-399, doi: 10.1097/ MOL.0000000000000112, indexed in Pubmed: 25137612.

10. Greenwald SE. Ageing of the conduit arteries. J Pathol. 2007; 211(2): 157-172, doi: 10.1002/path.2101, indexed in Pubmed: 17200940.

11. Stary HC, Chandler AB, Dinsmore RE, et al. A definition of advanced types of atherosclerotic lesions and a histological classification of atherosclerosis. A report from the Committee on Vascular Lesions of the Council on Arteriosclerosis, American Heart Association. Arterioscler Thromb Vasc Biol. 1995; 15(9): 1512-1531, doi: 10.1161/01.atv.15.9.1512, indexed in Pubmed: 7670967.

12. Stary HC, Blankenhorn DH, Chandler AB, et al. A definition of the intima of human arteries and of its atherosclerosis-prone regions. A report from the Committee on Vascular Lesions of the Council on Arteriosclerosis, American Heart Association. Arterioscler Thromb. 1992; 12(1): 120-134, doi: 10.1161/01.atv.12.1.120, indexed in Pubmed: 1731855.

13. Stary HC, Chandler AB, Glagov S, et al. A definition of initial, fatty streak, and intermediate lesions of atherosclerosis. A report from the Committee on Vascular Lesions of the Council on Arteriosclerosis, American Heart Association. 
Arterioscler Thromb. 1994; 14(5): 840-856, doi: 10.1161/01. atv.14.5.840, indexed in Pubmed: 8172861.

14. Järveläinen H, Sainio A, Koulu M, et al. Extracellular matrix molecules: potential targets in pharmacotherapy. Pharmacol Rev. 2009; 61(2): 198-223, doi: 10.1124/pr.109.001289, indexed in Pubmed: 19549927.

15. Duprez DA, Gross MD, Ix JH, et al. Collagen biomarkers predict new onset of hypertension in normotensive participants: the Multi-Ethnic Study of Atherosclerosis. J Hypertens. 2018; 36(11): 2245-2250, doi: 10.1097/HJH.0000000000001793, indexed in Pubmed: 29782392.

16. Wang H, Liu D, Zhang H. Investigation of the underlying genes and mechanism of macrophage-enriched ruptured atherosclerotic plaques using bioinformatics method. J Atheroscler Thromb. 2019; 26(7): 636-658, doi: 10.5551/jat.45963, indexed in Pubmed: 30643084.

17. Holm Nielsen S, Jonasson L, Kalogeropoulos K, et al. Exploring the role of extracellular matrix proteins to develop biomarkers of plaque vulnerability and outcome. J Intern Med. 2020; 287(5): 493-513, doi: 10.1111/joim.13034, indexed in Pubmed: 32012358.

18. Kuzan A, Chwiłkowska A, Maksymowicz K, et al. Advanced glycation end products as a source of artifacts in immunoenzymatic methods. Glycoconj J. 2018; 35(1): 95-103, doi: 10.1007/s10719-017-9805-4, indexed in Pubmed: 29305778.

19. Kuzan A, Chwiłkowska A, Pezowicz C, et al. The content of collagen type II in human arteries is correlated with the stage of atherosclerosis and calcification foci. Cardiovasc Pathol. 2017; 28: 21-27, doi: 10.1016/j.carpath.2017.02.003, indexed in Pubmed: 28284062.

20. Briones AM, Salaices M, Vila E. Mechanisms underlying hypertrophic remodeling and increased stiffness of mesenteric resistance arteries from aged rats. J Gerontol A Biol Sci Med Sci. 2007; 62(7): 696-706, doi: 10.1093/gerona/62.7.696, indexed in Pubmed: 17634315.

21. Aikawa M, Rabkin E, Okada Y, et al. Lipid lowering by diet reduces matrix metalloproteinase activity and increases collagen content of rabbit atheroma: a potential mechanism of lesion stabilization. Circulation. 1998; 97(24): 2433-2444, doi: 10.1161/01.cir.97.24.2433, indexed in Pubmed: 9641696.

22. Abdelhalim MA, Siddiqi NJ, Alhomida AS, et al. The changes in various hydroxyproline fractions in aortic tissue of rabbits are closely related to the progression of atherosclerosis. Lipids Health Dis. 2010; 9: 26, doi: 10.1186/1476-511X-9-26, indexed in Pubmed: 20214825.

23. Sakalihasan N, Heyeres A, Nusgens BV, et al. Modifications of the extracellular matrix of aneurysmal abdominal aortas as a function of their size. Eur J Vasc Surg. 1993; 7(6): 633-637, doi: 10.1016/s0950-821x(05)80708-x, indexed in Pubmed: 8270064.

24. Kong $\mathrm{CH}$, Lin $\mathrm{XY}$, WooCC, et al. Characteristics of aortic wall extracellular matrix in patients with acute myocardial infarction: tissue microarray detection of collagen I, collagen III and elastin levels. Interact Cardiovasc Thorac Surg. 2013; 16(1): 11-15, doi: 10.1093/icvts/ivs421, indexed in Pubmed: 23049084

25. Cantini C, Kieffer P, Corman B, et al. Aminoguanidine and aortic wall mechanics, structure, and composition in aged rats. Hypertension. 2001; 38(4): 943-948, doi: 10.1161/ hy1001.096211, indexed in Pubmed: 11641314.

26. Sindram D, Martin K, Meadows JP, et al. Collagen-elastin ratio predicts burst pressure of arterial seals created using a bipolar vessel sealing device in a porcine model. Surg Endosc. 2011; 25(8): 2604-2612, doi: 10.1007/s00464-011-1606-4, indexed in Pubmed: 21404086.
27. Katsuda S, Okada Y, Minamoto T, et al. Collagens in human atherosclerosis. Immunohistochemical analysis using collagen type-specific antibodies. Arterioscler Thromb. 1992; 12(4): 494-502, doi: 10.1161/01.atv.12.4.494, indexed in Pubmed: 1373075.

28. Shekhonin BV, Domogatsky SP, Idelson GL, et al. Relative distribution of fibronectin and type I, III, IV, V collagens in normal and atherosclerotic intima of human arteries. Atherosclerosis. 1987; 67(1): 9-16, doi: 10.1016/0021-9150(87)902590, indexed in Pubmed: 3314885.

29. Jing $\mathrm{L}, \mathrm{Li} \mathrm{L}, \mathrm{Ren} \mathrm{X}$, et al. Role of sortilin and matrix vesicles in Ne-carboxymethyl-lysine-induced diabetic atherosclerotic calcification. Diabetes Metab Syndr Obes. 2020; 13: 4141-4151, doi: 10.2147/DMSO.S273029, indexed in Pubmed: 33177854.

30. Tsai CH, Lin LY, Lin YH, et al. Abdominal aorta calcification predicts cardiovascular but not non-cardiovascular outcome in patients receiving peritoneal dialysis: A prospective cohort study. Medicine (Baltimore). 2020; 99(37): e21730, doi: 10.1097/MD.0000000000021730, indexed in Pubmed: 32925715.

31. Kuga T, Esato K, Zempo N, et al. Detection of type III collagen fragments in specimens of abdominal aortic aneurysms. Surg Today. 1998; 28(4): 385-390, doi: 10.1007/ s005950050146, indexed in Pubmed: 9590702.

32. Lui PPY, Chan LS, Lee YW, et al. Sustained expression of proteoglycans and collagen type III/type I ratio in a calcified tendinopathy model. Rheumatology (Oxford). 2010; 49(2): 231-239, doi: 10.1093/rheumatology/kep384, indexed in Pubmed: 19955224

33. Volk SW, Shah SR, Cohen AJ, et al. Type III collagen regulates osteoblastogenesis and the quantity of trabecular bone. Calcif Tissue Int. 2014; 94(6): 621-631, doi: 10.1007/s00223014-9843-x, indexed in Pubmed: 24626604.

34. Asgari M, Latifi N, Heris HK, et al. In vitro fibrillogenesis of tropocollagen type III in collagen type I affects its relative fibrillar topology and mechanics. Sci Rep. 2017; 7(1): 1392, doi: 10.1038/s41598-017-01476-y, indexed in Pubmed: 28469139.

35. Busch A, Hoffjan S, Bergmann F, et al. Vascular type Ehlers-Danlos syndrome is associated with platelet dysfunction and low vitamin D serum concentration. Orphanet J Rare Dis. 2016; 11(1): 111, doi: 10.1186/s13023-016-0491-2, indexed in Pubmed: 27488172.

36. Stary HC, Chandler AB, Dinsmore RE, et al. A definition of advanced types of atherosclerotic lesions and a histological classification of atherosclerosis. A report from the Committee on Vascular Lesions of the Council on Arteriosclerosis, American Heart Association. Circulation. 1995; 92(5): 1355-1374, doi: 10.1161/01.cir.92.5.1355, indexed in Pubmed: 7648691.

37. Lee JS, Basalyga DM, Simionescu A, et al. Elastin calcification in the rat subdermal model is accompanied by up-regulation of degradative and osteogenic cellular responses. Am J Pathol. 2006; 168(2): 490-498, doi: 10.2353/ajpath.2006.050338, indexed in Pubmed: 16436663.

38. Qin X, Corriere MA, Matrisian LM, et al. Matrix metalloproteinase inhibition attenuates aortic calcification. Arterioscler Thromb Vasc Biol. 2006; 26(7): 1510-1516, doi: 10.1161/01. ATV.0000225807.76419.a7, indexed in Pubmed: 16690876.

39. Pereira L, Lee SY, Gayraud B, et al. Pathogenetic sequence for aneurysm revealed in mice underexpressing fibrillin-1. Proc Natl Acad Sci U S A. 1999; 96(7): 3819-3823, doi: 10.1073/pnas.96.7.3819, indexed in Pubmed: 10097121.

40. Bailey M, Pillarisetti S, Jones P, et al. Involvement of matrix metalloproteinases and tenascin- $\mathrm{C}$ in elastin calcification. Cardiovasc Pathol. 2004; 13(3): 146-155, doi: 10.1016/S10548807(04)00009-2, indexed in Pubmed: 15081471. 
41. Reimann C, Brangsch J, Colletini F, et al. Molecular imaging of the extracellular matrix in the context of atherosclerosis. Adv Drug Deliv Rev. 2017; 113: 49-60, doi: 10.1016/j. addr.2016.09.005, indexed in Pubmed: 27639968.

42. Chen W, Cormode DP, Vengrenyuk Y, et al. Collagen-specific peptide conjugated HDL nanoparticles as MRI contrast agent to evaluate compositional changes in atherosclerotic plaque regression. JACC Cardiovasc Imaging. 2013; 6(3): 373-384, doi: 10.1016/j.jcmg.2012.06.016, indexed in Pubmed: 23433925.

43. Kassam HA, Bahnson EM, Cartaya A, et al. Pharmacokinetics and biodistribution of a collagen-targeted peptide amphiphile for cardiovascular applications. Pharmacol Res Perspect. 2020; 8(6): e00672, doi: 10.1002/prp2.672, indexed in Pubmed: 33090704.

44. Zheng J, Li Q, He L, et al. Protocatechuic Acid Inhibits Vulnerable Atherosclerotic Lesion Progression in Older Apoe/- Mice. J Nutr. 2020; 150(5): 1167-1177, doi: 10.1093/jn/ nxaa017, indexed in Pubmed: 32047914.

45. Tomosugi N, Yamamoto S, Takeuchi M, et al. Effect of Collagen Tripeptide on Atherosclerosis in Healthy Humans. J Atheroscler Thromb. 2017; 24(5): 530-538, doi: 10.5551/ jat.36293, indexed in Pubmed: 27725401.

Submitted: 15 October, 2019 Accepted after reviews: 28 January, 2021 Available as AoP: 8 February, 2021 